flux density, $d$ is the distance to the source, and $B_{\nu}$ is the blackbody Planck intensity. We can improve on this estimate by using a model $^{14,15}$ in which radial disk temperature and density are allowed to vary as power laws. The exponent, 0.61 , of the radial profile in disk temperature is obtained from a least-squares fit to the infrared spectral energy distribution; the radial density exponent ${ }^{15}$ is set at $7 /$ 4 . Free parameters are disk mass, $M_{\mathrm{D}}$, and $\beta$. From model fits to our 1.3- and 2.7-mm flux densities measured with the millimetre-wave array and to single-dish sub-millimetre flux densities (V. M. et al., manuscript in preparation), we derive best-fit values for $M_{\mathrm{D}}$ and $\beta$ of $(0.05 \pm 0.01) M_{\odot}$ and $1.0 \pm 0.2$, respectively. We conclude that the true disk mass is a few per cent of a solar mass rather than the very low value derived from the $\mathrm{CO}$ line intensity. The opacity index, $\beta$, is significantly less than the value of 2 normally adopted ${ }^{16}$ for dust grains in the interstellar medium (ISM). This could be due to a population of grains in the MWC480 disk that are larger than $1 \mathrm{~mm}$ in size ${ }^{17}$, that is, several orders of magnitude greater than the sizes of ISM grains. Although this raises the possibility of grain accumulation, perhaps associated with the formation of larger bodies such as planetesimals, we note that opacity indices are also sensitive to chemical composition ${ }^{8}$ and to grain morphology ${ }^{19}$.

At $2.3 M_{\odot}$, the MWC480 stellar mass is a factor of 3 greater than the typical value for T Tauri stars ${ }^{20}$ (TTs). The TTs are young premain-sequence stars with later spectral types than the Herbig Ae stars (typically $\mathrm{Me}$ and $\mathrm{Ke}$ ) and concomitantly lower masses, $\sim 0.7 M_{\odot}$ on average ${ }^{20}$. Millimetre-wave aperture synthesis images and kinematic models ${ }^{21,22}$ confirm that, as suggested by photometric observations ${ }^{20}$, many TTs are accompanied by circumstellar disks that could be protoplanetary in nature ${ }^{23,24}$. Our new observations of MWC480 provide the strongest evidence to date for a rotating disk around a higher-mass counterpart to the TTs.

The mass of the MWC480 disk, $(0.02-0.05) M_{\odot}$, is close to the peak of the mass distribution measured for disks of $\mathrm{TTs}^{20}$. By contrast, disks around Vega-like stars are much less massive, by factors ${ }^{1}$ of $10^{-5}$ to $10^{-6}$. And, whereas the MWC480 and TT disks are dominated by gas, the Vega-like disks appear to be composed predominantly of grains ${ }^{25}$. Indeed, the ages of Vega-like stars greatly exceed the timescales, in the absence of gas, for the removal of small inner-disk grains via radiation pressure and Poynting-Robertson drag. This has prompted suggestions ${ }^{1,26}$ that the grains are replenished by debris from continuing collisions and/or disruption of planetesimals. Could the MWC480 system represent the early stages of such a debris disk? With an age of $6 \mathrm{Myr}$, the massive rotating disk is a dense reservoir of orbiting material that could be sufficiently long-lived to support the growth of planetesimals. In fact the age of the disk exceeds, by two to three orders of magnitude, current estimates of the mean formation timescale ${ }^{5,27}$ for kilometre-sized planetesimals. Moreover, the mass of the MWC480 disk is greater than the minimum ${ }^{5}$ required to build a planetary system with an aggregate mass comparable to that of our Solar System. The disk encircling MWC480 could well be a progenitor of a debris disk such as that surrounding Pic, and provides an opportunity to examine the processes by which planetary systems are created.

Received 28 April; accepted 17 June 1997

. Backman, D. E. \& Paresce, F. in Protostars \& Planets III (eds Levy, E. H. \& Lunine, J.) 1253-1304 (Univ. Arizona Press, Tucson, 1993).

2. Smith, B. A. \& Terrile, R. J. A circumstellar disk around $\beta$ Pictoris. Science 226, 1421-1424 (1984). Golimowski, D. A., Durrance, S. T. \& Clampin, M. Coronagraphic imaging of the $\beta$ Pictoris circumstellar disk: evidence of changing disk structure within 100 AU. Astrophys. J. 411, L41-L44 (1993),

4. Lagage, P. O. \& Pantin, E. Dust depletion in the inner disk of $\beta$ Pictoris as a possible indicator of planets. Nature 369, 628-630 (1994)

Lissauer, J. J. Planet formation. Annu. Rev. Astron. Astrophys. 31, 129-174 (1993).

6. Mannings, V. \& Sargent, A. I. A high-resolution study of gas and dust around young intermediatemass stars: evidence for circumstellar disks in Herbig Ae systems. Astrophys. J. (in the press).

. Herbig, G. H. The spectra of Be- and Ae-type stars associated with nebulosity. Astrophys. J. Suppl. Ser. 4, 337-368 (1960)

. Aumann, H. H. et al. Discovery of a shell around $\alpha$ Lyr. Astrophys. J. 278, L23-L27 (1984).

9. Sylvester, R. J., Barlow, M. J., Skinner, C. J. \& Mannings, V. Optical, infrared and millimetre-wave properties of Vega-like systems. Mon. Not. R. Astron. Soc. 279, 915-939 (1996)

10. D’Antona, F. \& Mazzitelli, I. New pre-main-sequence tracks for $M \leq 2.5 M_{\odot}$ as tests of opacities and convection model. Astrophys. J. Suppl. Ser. 90, 467-500 (1994).
11. Koerner, D. W. in CO: Twenty-Five Years of Millimeter-Wave Spectroscopy (eds Latter, W. B. et al.) 162164 (IAU Symp. 170, Kluwer, Dordrecht, 1997).

2. Scoville, N. Z. et al. High-resolution mapping of molecular outflows in GGC 2071, W49, and NGC 7538. Astrophys. J. 303, 416-432 (1986).

13. Hildebrand, R. H. Determination of cloud masses and dust characteristics from submillimetre thermal emission. Q. J. R. Astron. Soc. 24, 267-282 (1983).

14. Beckwith, S. V. W. \& Sargent, A. I. Particle emissivity in circumstellar disks. Astrophys. J. 381, 250-258 (1991)

15. Mannings, V. \& Emerson, J. P. Dust in disks around T Tauri stars: grain growth? Mon. Not. R. Astron. Soc. 267, 361-378 (1994)

16. Draine, B. T. \& Lee, H. M. Optical properties of interstellar graphite and silicate grains. Astrophys. J. 285, 89-108 (1984).

17. Miyake, K. \& Nakagawa, Y. Effects of particle size distribution on opacity curves of protoplanetary disks around T Tauri stars. Icarus 106, 20-41 (1993).

18. Pollack, J. B. et al. Composition and radiative properties of grains in molecular clouds and accretion disks. Astrophys. J. 421, 615-639 (1994).

19. Wright, E. L. Long-wavelength absorption by fractal dust grains. Astrophys. J. 320, 818-824 (1987). 20. Beckwith, S. V. W., Sargent, A. I., Chini, R. S. \& Güsten, R. A survey for circumstellar disks around young stellar objects. Astron. J. 99, 924-945 (1990).

21. Koerner, D. W., Sargent, A. I. \& Beckwith, S. V. W. A rotating gaseous disk around the T Tauri star GM Aurigae. Icarus 106, 2-10 (1993).

22. Dutrey, A., Guilloteau, S. \& Simon, M. Images of the GG Tauri rotating ring. Astron. Astrophys. 286, 149-159 (1994).

23. Beckwith, S. V. W. \& Sargent, A. I. Circumstellar disks and the search for neighbouring planetary systems. Nature 383, 139-144 (1996).

24. Sargent, A. I. in Disks \& Outflows around Young Stars (eds Beckwith, S. V. W., Staude, J., Quetz, A. \& Natta, A.) 1-23 (Springer, Berlin, 1996)

25. Dent, W. R. F., Greaves, J. S., Mannings, V., Coulson, I. M. \& Walther, D. M. A search for molecular gas components in prototypal Vega-excess systems. Mon. Not. R. Astron. Soc. 277, L25-L29 (1995).

26. Beust, H. \& Lissauer, J. J. The effects of stellar rotation on the absorption spectra of comets orbiting $\beta$ Pictoris. Astron. Astrophys. 282, 804-810 (1994).

27. Weidenschilling, S. The origin of comets in the solar nebula: a unified model. Icarus (in the press)

Acknowledgements. V.M. thanks M. Romans and N. Romans for their support. We thank the staff at the Owens Valley Radio Observatory for their assistance. The Owens Valley millimetre-wave array is supported by the NSF; array studies of young star and disk systems are supported in part by the Norris Planetary Origins project and by NASA's Origins of Solar Systems programme.

Correspondence should be addressed to V.M. (e-mail: vgm@astro.caltech.edu).

\section{Observation of an internal wave attractor in a confined, stably stratified fluid}

\section{Leo R. M. Maas ${ }^{\star}$, Dominique Benielli $\dagger$, Joël Sommeria $\nmid$ \& Frans-Peter A. Lam*}

* Netherlands Institute for Sea Research, PO Box 59, 1790 AB Texel,

The Netherlands

$\dagger$ Ecole Normale et Superieure de Lyon, Laboratoire de Physique, 46 allée d'Italie, 69364 Lyon cedex 07, France

When a container of water is vibrated, its response can be described in terms of large-scale standing waves-the eigenmodes of the system. The belief that enclosed continuous media always possess eigenmodes is deeply rooted. Internal gravity waves in uniformly stratified fluids, however, present a counterexample. Such waves propagate at a fixed angle to the vertical that is determined solely by the forcing frequency, and a sloping side wall of the container will therefore act as a lens, resulting in ray convergence or divergence. An important consequence of this geometric focusing is the prediction ${ }^{1}$ that, following multiple reflections, these waves will evolve onto specific paths - or attractors-whose locations are determined only by the frequency. Here we report the results of laboratory experiments that confirm that internal-wave attractors, rather than eigenmodes, determine the response of a confined, stably stratified fluid over a broad range of vibration frequencies. The existence of such attractors could be important for mixing processes in ocean basins and lakes, and may be useful for analysing oscillations of the Earth's liquid core and the stability of spinning, fluid-filled spacecraft.

The density gradient in a fluid is mainly determined by its temperature and dissolved salts. When the density increases at a constant rate in the direction of gravity the fluid, assumed to be 
incompressible, is stably stratified and is characterized by a constant value of the stability frequency $N$. Regular perturbations of this static equilibrium, of frequency $\omega<N$, lead to internal gravity waves whose energy propagates obliquely ${ }^{2}$ along straight lines-the rays, that make a fixed angle $\theta=\arccos (\omega / N)$ with the vertical. In our experiments, a uniformly stratified fluid is contained in a narrow tank, confining the wave propagation to a vertical plane. Tracing the rays that bounce back and forth between top and bottom boundaries and side walls is like following a frictionless ball on a billiard table, except that in this case the angle of reflection no longer needs to equal the angle of incidence as the 'ball' can only move in one of four directions, $\pm \theta(\bmod \pi)$, relative to the vertical. In a rectangular domain, whose boundaries are horizontal and vertical, there is no difference with the classical billiard table. Provided the depth-to-width ratio is rational, any ray travels along a closed path of finite length ${ }^{1}$ and two rays retain their distance apart. In contrast, when one of the walls is sloping, two incident rays get focused or defocused on reflection from it (Fig. 1A). The case when these two processes offset each other, and all orbits are again closed (a 'global resonance'), is exceptional. Also, chaotic rays, encountered in a variety of classical billiards ${ }^{3}$, are never obtained. Instead, in most cases focusing dominates and all ray paths become infinitely long, while approaching an attractor $^{1}$. An example is the $(1,1)$-attractor in Fig. 1A, the

A

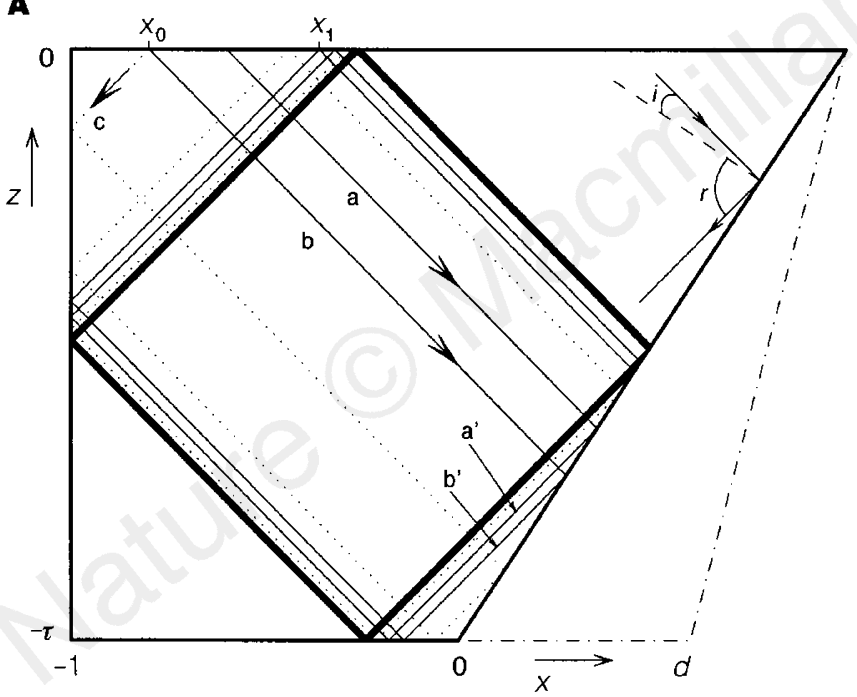

square of heavy black lines, where an $(m, n)$-attractor is defined as having $m$ reflections at the surface and $n$ reflections at the vertical side-wall.

For a time-periodic disturbance, the spatial structure of the internal waves is a solution of a linear, hyperbolic (wave) equation in terms of the pressure and streamfunction fields ${ }^{2}$, which, in turn, determine the velocity and density fields (Fig. 1B). This equation has solutions that are constant along lines, so-called characteristics, which coincide with the rays. Obtaining the ray paths - 'webs' of connected characteristics - is therefore the first, geometric step in solving the hyperbolic equation. They span the fluid domain in a complete, but non-uniform way, as each of the rays approaches the attractor. Ray geometry shows that for any given frequency, the fluid domain is endowed with a structure (ultimately characterized by the shape of its attractor). The second step involved in solving this equation is contained in a description of the 'dynamics' on these webs, which visualizes ('dresses') this structure. Here, this amounts to the prescription of a 'partial pressure' $f$ on each web, a quantity that is conserved along it. Pressure and streamfunction fields at an arbitrary point are then simply given by the sum and differences of the partial-pressure fields on the two characteristics that go through that point, and have a geometrical pattern that reproduces itself at smaller scales (Fig. 1B). The spatial structure is self-similar (fractal), and so is the dependence of the solution on dimensionless depth $\tau$

B
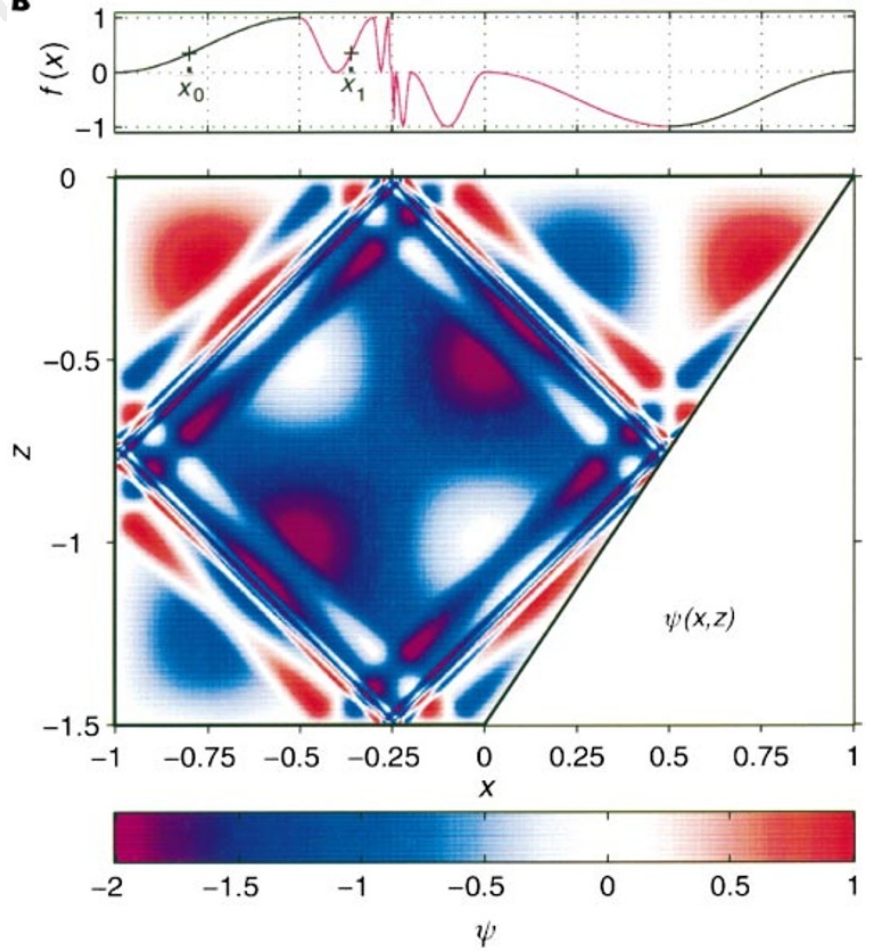

Figure 1 A, Sketch of basin with sloping side wall, varying in shape from triangular $(d=-1)$ to rectangular $(d=+1)$. Gravity acts vertically downwards. The general shape $(d \neq 0)$ is dash-dotted, whereas the case $d=0$, considered in the experiments, is given by solid lines. Coordinates $x$ and $z$ are scaled with half-width $L$ and $L /|\tan \theta|$ respectively, so that true depth $D$ is stretched to dimensionless depth $\tau=|\tan \theta| D / L=\left(N^{2} / \omega^{2}-1\right)^{1 / 2} D / L \quad(\tau=3 / 2$ in this diagram $)$. With this scaling, waves always propagate with an apparent inclination of $45^{\circ}$. Angle of reflection $r$ on a sloping wall (with respect to its normal) therefore differs from angle of incidence $i$. When $r>i$, two parallel rays a and b, bouncing on a sloping wall, remain parallel after reflection, but get focused to $a^{\prime}$ and $b^{\prime}$, eventually converging on the attractor, the square of heavy black lines. Defocusing is obtained, when $i>r$, as for rays $a^{\prime}$ and $b^{\prime}$ (reversing their direction), even though these rays eventually also approach the attractor, as ray c (dotted line), initially in a direction opposite to $\mathrm{b}$, shows. Surface intersections $x_{0}, x_{1}, \ldots$ show convergence (to $x=-0.25)$. B, The spatial part of the streamfunction $\psi(x, z)$ exp $(i \omega t)$, as governed by the hyperbolic equation $\psi_{x x}-\psi_{z z}=0$, subject to the vanishing of $\psi$ at the boundary (the impermeability condition). Here subscripts denote partial derivatives. A solution, vanishing at $z=0$, is $\psi(x, z)=f(x-z)-f(x+z)$. The solution must also vanish at the other boundaries, which is satisfied when partial pressure $f$ is invariant on each web of characteristics (lines $x \pm z=$ constant) ${ }^{1,21}$. Then we can find $\psi(x, z)$ provided the value of $f(x)$ is prescribed on some appropriate intervals, as for the black line in the top panel within $x$ intervals $[-1,-0.5]$ and $[0.5,1]$. Starting, for instance, on the upper boundary at $x_{0}$ in the interval $[-1,-0.5]$, following connected characteristics, we return to $x_{1}$, in the neighbouring interval $[-0.5,-0.3]$, and infer $f\left(x_{1}\right)=f\left(x_{0}\right)$. By iterating this process for all points within these intervals we obtain $f$ at each characteristic and hence the streamfunction. 
(related to the wave period) and on the geometric parameter $d$ (Fig. 2; these quantities are defined in Fig. 1).

The equation determining the spatial structure of the usual standing waves (for example, surface, or interfacial waves) is elliptic rather than hyperbolic and reduces to a standard eigenvalue problem. Solutions - eigenmodes, or seiches ${ }^{4}$ — are then obtained only for a set of resonance frequencies, and they are smooth. These modes also dominate the response for arbitrary forcing frequencies. Here by contrast, we get solutions at any frequency below $N$ and solutions are not smooth along the attractor (they are singular). For this reason the problem is often classified as 'ill-posed". These solutions are still quite relevant physically, as shown by the laboratory experiments below.

A rectangular plexiglass container was filled with an exponentially stratified salt solution, such that the stability frequency was constant at $N=1.89 \mathrm{~s}^{-1}$. The container (shown in Fig. 3a) had a sloping side wall, extending from bottom centre to top right-hand corner yielding $d=0$. Overall dimensions were: width $(W) 96 \mathrm{~mm}$; depth $(D) 261 \mathrm{~mm}$; and length $(2 L) 261 \mathrm{~mm}$. Internal waves are visualized by the displacement of dye bands. These bands are produced by periodic dye injection into the brine entering the bottom of the tank, marking fluid parcels of given density. The deformation of the dye strips is visualized by the fluorescence excited by a vertical laser sheet.
The tank oscillates vertically with frequency $2 \omega$ (and amplitude $Z$ ), so as to produce a modulation of gravity (with amplitude $4 Z \omega^{2}$ ). Any wave with frequency $\omega$ is then parametrically amplified, as for a pendulum. The waves are everywhere excited with the same phase, set by the forcing. The temporal evolution is therefore separable from the spatial structure, described by the hyperbolic equation. However, the amplitude is now growing exponentially in time, according to the canonical Mathieu equation, describing parametric excitation ${ }^{6}$.

Because of viscous effects, small-scale attractors are likely to be more suppressed, so our attempts were directed towards observing large-scale attractors. For the geometry described above, one expects to find a $(1,1)$-attractor in the interval of periods $1 \leqslant \tau \leqslant 2$, or, for the adopted aspect ratio $D / L=2$, in the frequency interval $1 / \sqrt{2}<\omega / N<2 / \sqrt{5}$.

We therefore explore this frequency range (keeping a constant excitation amplitude $Z=10 \mathrm{~cm}$ ). Approximately five minutes after the oscillation is started, a two-dimensional oscillatory motion of the dye lines with frequency $\omega$ becomes visible. This oscillation is localized, takes on a box-shaped form, and appears most pronounced around the predicted location of the $(1,1)$-attractor (Fig. $3 a)$. In this initial growth phase, the oscillation has a standing nature: all points move up and down at the same time, while gradually growing in amplitude. Traces of strong shearing motion

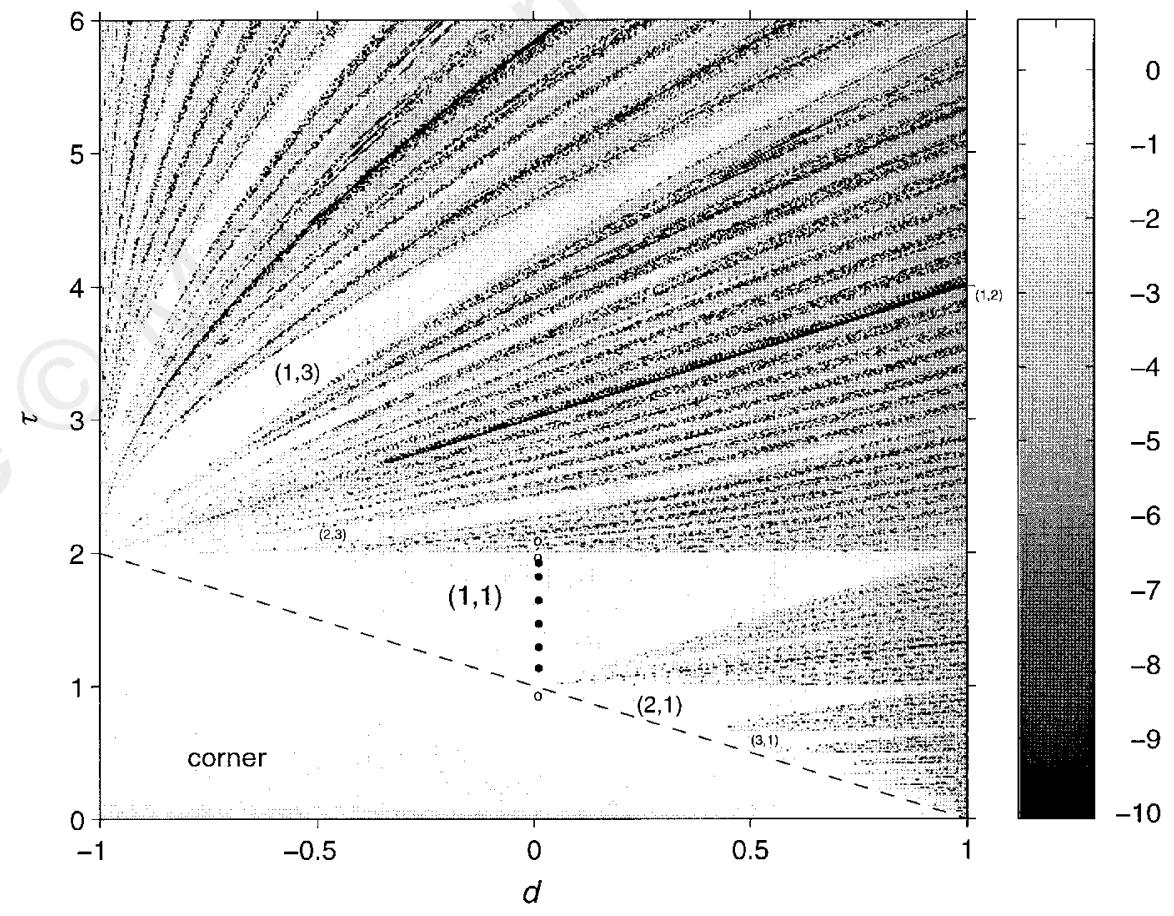

Figure 2 Convergence rate of neighbouring wave rays in parameter plane spanned by non-dimensional depth $\tau$ (related to internal wave period) and geometric parameterd (see definitions in Fig.1A). As wave rays eventually almost always converge, the Lyapunov exponent $\lambda$, measuring this convergence, is generally negative and $\log _{10}(-\lambda)$ is therefore displayed. Light (dark) regions are strongly (weakly) convergent. Regions and lines are labelled with integer pairs ( $m$, $n)$, which describe the number of reflections at the surface $(m)$ and vertical side wall $(n)$ of the attractor, or global resonance. For an $(m, n)$-attractor only one ray (the attractor) is truly periodic, for a global resonance every ray is. Continuous, light-coloured regions, identifying large-scale attractors (low $m$ and $n$ ), are separated by darker regions where fine-scale attractors reign. Within these ranges still finer-scale attractors reside, ultimately approaching the black lines for which there is no convergence at all (zero Lyapunov exponent): the set of global resonances. Wave rays may also be attracted to the right-hand upper corner of the basin in Fig. 1A, but this occurs only when the bottom slope is less steep than the rays (a subcritical slope) ${ }^{22}$, below the dashed line $\tau=1-d$. Above the critical curve, $(m, n)$-attractors can be identified. Remarkably, only attractors with an odd number of 'vertical' cells $n$, appear. The 'missing', even-numbered cells, correspond to the $(m, n)$-global resonances. The first of these, the $(1,2)$-global resonance, is clearly identified by the black line $\tau=3+d$. Note that $(m, n)$ attractors, as well as $(m, n)$-global resonances, approach the classical $(m, n)$ cellular solutions of the form $\psi=\sin [m \pi(x+1) / 2] \sin [n \pi z / \tau]$ of the rectangle, which are found at $\tau=2 n / m$, as $d \rightarrow 1$. Filled and open circles at $d=0$ indicate laboratory experiments in which 'focusing modes' were, or were not observed. 
can be detected by the occurrence of sharp gradients in dye concentrations, especially near the part of the attractor closest to the sloping side wall (where the focusing takes place). Dye mixing is later observed in this zone.

This structure contrasts strongly with the resonance modes previously observed with the same apparatus ${ }^{7}$ in a rectangular geometry (Fig. 3b). Moreover, the wave attractor changes smoothly with frequency, whereas the resonance mode was obtained only in a narrow frequency range (depending on the forcing amplitude, as an instability 'tongue'). Instead, the attractor is observed in the whole predicted frequency interval, as indicated by filled circles in Fig. 2. Outside this interval (open circles), we observe no wave excitation, or complex (unidentified) three-dimensional modes.

Visualization of the attractor can be improved by subtracting the initial state (where dye lines are flat), as in Fig. 4a for the standing

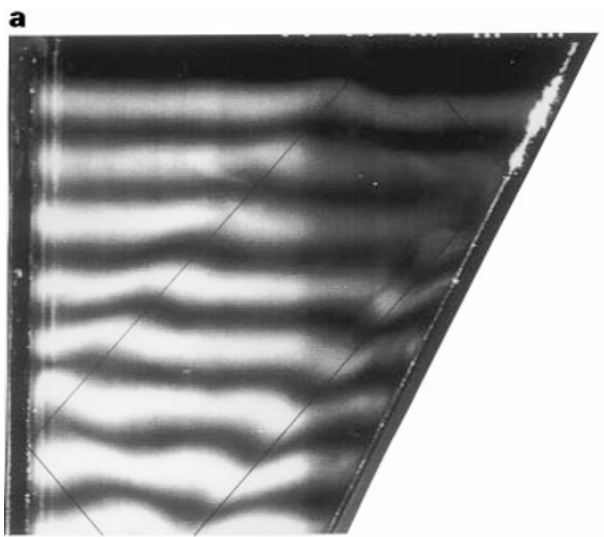

b

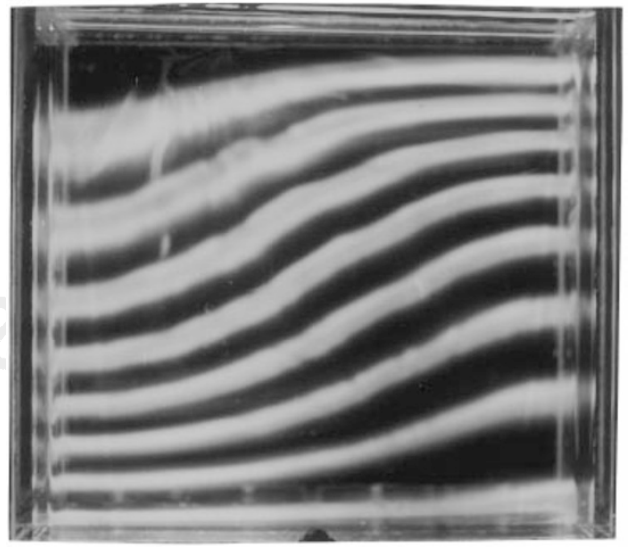

Figure $\mathbf{3}$ a, Side view of laboratory tank, showing a maximum displacement of (initially horizontal) dye lines during the growth phase (standing wave), 9 minutes after the oscillation of the table was started. As expected, the displacement is strongest along the attractor, represented by the solid line (tank oscillation amplitude $Z=10 \mathrm{~cm}, \tau=1.71$ ). Note that the bottom of the tank is located slightly below the lower edge of the figure. The occurrence of wave growth depends on the frequency and amplitude of excitation and is apparently inhibited by viscous effects when the attractor shape becomes either too complicated, or too narrow, as when the box-shaped attractor degenerates into a line. Thus for this tank $(d=0)$, in the frequency interval for which the $(1,1)$-attractor is obtained, the instability threshold is close to $Z \approx 15 \mathrm{~cm}$ near the bounds of the interval, but is lower, $Z \approx 8 \mathrm{~cm}$ at its middle. $\mathbf{b}$. As $\mathbf{a}$, but with a square domain (from ref. 7), for which an eigenmode structure is obtained, in sharp contrast with the attractor observed in the present study. phase. After about two minutes, the standing wave gives way to the permanent regime, in which the amplitude of the internal wave saturates. The wave then takes on a propagating character (Fig. 4b), while it displays some chaotic particle motion. Phase propagation is perpendicular to the attractor, as clearly observed by tracking a wave nodal line (having zero elevation); this is consistent with an energy flux directed parallel to the attractor, in the clockwise (focusing) direction.

The concurrently observed irregular behaviour near the slope could be related to chaotic particle transport ${ }^{8}$ by the propagating wave. The standing-wave regime by contrast is very regular. Particle motion is then periodic, the streamfunction acting like the hamiltonian of a system with one degree of freedom.

A stratified fluid in a non-trivially shaped container possesses inviscid modes of oscillation which are singular along a wave

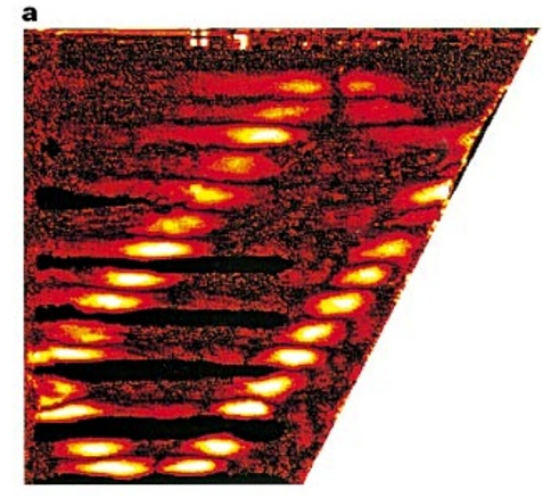

b
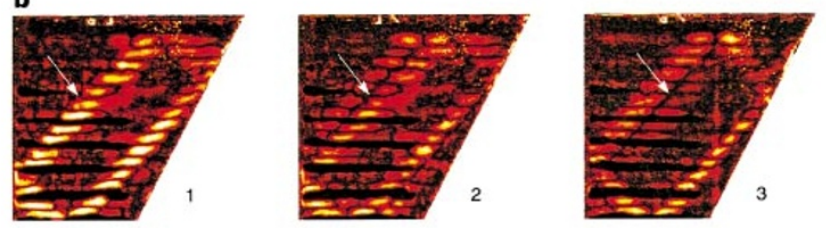

Figure 4 a, Side view of tank showing the difference between (maximally) displaced dye lines and their horizontal initial state (using computer processing) in the standing phase, 9 minutes after oscillation of the tank was started $(Z=10 \mathrm{~cm}, \tau=1.74)$. b. As $\mathbf{a}$, but in the permanent regime (10 minutes after the start of oscillation) showing a propagating wave. Energy propagation of internal gravity waves is perpendicular to phase propagation (such that their vertical components are opposite). Thus, in Fig. 1A, when phase propagates from b to a, energy propagates along these phase-lines, in the direction of the arrows. In the permanent regime, phase propagation perpendicular to the attractor is observed on each of the four sides of the box-shaped attractor in a manner consistent with clockwise energy propagation around it. This is seen by tracking (black) nodal lines on successive views (indicated by arrows on one side of the box). Integer labels $n$ (where $n=1,2$ and 3 ) refer to relative time $T \times n / 12$, where $T=4.37 \mathrm{~s}$ is the wave period. Propagating waves are theoretically obtained for complex $f$. For each solution, there is also a complex conjugate solution, representing a wave propagating in the opposite direction. Each wave has an infinite path, and can be likened to waves on an infinite string. But both waves approach the same attractor, as illustrated by rays $b$ and $c$ in Fig. 1A. In the growth phase these oppositely propagating waves combine to form a standing wave, whose uniform phase allows effective growth by parametric instability. Once these waves reach the vicinity of the attractor they transfer energy to smaller scales where it is selectively withdrawn by nonlinear and viscous processes. This provides the additional damping required for saturation of the parametric instability and breaks the symmetric propagation of energy through the 'string', thus giving the final wave pattern its propagating character. 
attractor. Our experiments confirm the physical relevance of this attractor, in spite of viscous and nonlinear effects. A standing wave is initially excited by the parametric instability, turning into a propagating wave in the permanent regime. A usual boundary forcing should only produce the latter regime, with permanent excitation at large scale, and energy transfers towards small scales along the attractor ${ }^{9}$. The system also has a broad-band response in frequency, set by the interval of existence of the attractor. This behaviour contrasts dramatically with the narrow peaks of usual resonances, whose width is set by dissipative effects.

Similar internal wave attractors could be predicted, by ray tracing, in ocean basins or lakes. Waves must be amplified by focusing at their contact with the sloping bottom. This should determine zones of intense mixing, favouring life, by providing a pathway for the transport of nutrients and rare metals from the bottom to the photic zone $\mathrm{e}^{10,11}$. This focusing process has often been invoked $^{12-14}$, but without considering its localization on a global attractor. Such localization depends of course on the wave frequency. In the ocean, important contributions at the tidal frequency should be expected, but, unlike our experiments, wind-related frequencies are simultaneously excited, and the widest frequency 'windows', corresponding to an attractor related to the particular geometry, may show up. The dressing of the attractor by the wave solution is clearly understood in our two-dimensional case, but a (non-trivial) three-dimensional generalization must be sought for natural basins.

Our results could also be relevant to an experiment ${ }^{15}$ that showed inertial oscillations in a fluid contained in a truncated cone rotating around its axis (where axisymmetric modes are excited by a sinusoidal modulation of the rotation rate). The spectral response was in this case interpreted in terms of resonance peaks of standing waves (presumably broadened by viscous effects). We note, however, that the spectral 'peaks' and 'valleys' coincide remarkably well with the regions of strong and weak convergence rates (obtained as an appropriate cross-section in Fig. 2 for $d=-0.478$ ): so we are led to conclude that the broadness of these response 'peaks' may well reflect a genuine, finite bandwidth of the response rather than a viscously spread-out resonance spike.

The problem of confined inviscid inertial oscillations, as occur in a spherical shell, is also ill-posed ${ }^{16}$ (unlike in a sphere). Smooth eigenmodes can be computed by introducing a small viscosity ${ }^{17}$, but better physical insight is expected in terms of wave attractors. The simplest attractors would again show up as wide resonance bands, whose width is independent of dissipative effects. This may be relevant to analysing inertial oscillations of the Earth core ${ }^{18,19}$ and to the stability of liquid-filled, rotating spacecraft ${ }^{20}$.

Received 19 December 1996; accepted 17 June 1997

1. Maas, L. R. M. \& Lam, F.-P. A. Geometric focusing of internal waves. J. Fluid Mech. 300, 1-41 (1995)

2. Görtler, H. Über eine Schwingungserscheinung in Flüssigkeiten mit stabiler Dichteschichtung. $Z$. Angew. Math. Mech. 23, 65-71 (1943).

Berry, M. V. Regularity and chaos in classical mechanics, illustrated by three deformations of a circular 'billiard'. Eur. J. Phys. 2, 91-102 (1981).

4. Wedderburn, E. M. The temperature seiche. I-IV. Phil. Trans. R. Soc. Edinb. 47, 619-642 (1911)

5. Courant, R. \& Hilbert, D. Methoden der Mathematischen Physik Vol. II (Springer, Berlin, 1937).

6. McEwan, A. D. \& Robinson, R. M. Parametric instability of internal gravity waves. J. Fluid Mech. 67, 667-687 (1975).

Benielli, D. \& Sommeria, J. Excitation of internal waves and stratified turbulence by parametric instability. Dyn. Atmos. Oceans 23, 335-343 (1996).

8. Joseph, B. Chaotic mixing by internal inertia-gravity waves. Phys. Fluids 9, 945-962 (1997).

9. Phillips, O. M. Energy transfer in rotating fluids by reflection of inertial waves. Phys. Fluids 6, 513-520 (1963).

10. Steele, J. H. Spatial Pattern in Plankton Communities (Plenum, New York, 1978).

11. Holligan, P. M., Pingree, R. D. \& Mardell, G. T. Oceanic solitons, nutrient pulses and phytoplankton growth. Nature 314, 348-350 (1985).

12. Eriksen, C. Implications of ocean bottom reflections for internal wave spectra and mixing. J. Phys. Oceanogr. 15, 1145-1156 (1985).

13. Garrett, C. J. R. \& Gilbert, D. in Small-scale Turbulence and Mixing in the Ocean (eds Nihoul, J. C. J. \& Jamart, B. M.) 405-423 (Elsevier, Amsterdam, 1988).

14. Ivey, G. N. \& Nokes, R. I. Vertical mixing due to the breaking of critical internal waves on sloping boundaries. J. Fluid. Mech. 204, 479-500 (1989).

15. Beardsley, R. C. An experimental study of inertial waves in a closed cone. Stud. Appl. Math. 49, 187196 (1970)

16. Stewartson, K. \& Rickard, J. A. Pathological oscillations of a rotating fluid. J. Fluid Mech. 35, 759-773 (1969).
17. Rieutord, M. Inertial modes in the liquid core of the earth. Phys. Earth Planet. Inter. 91, 41-46 (1995). 18. Melchior, P. \& Ducarme, B. Detection of inertial gravity oscillations in the Earth's core with a superconducting gravimeter at Brussels. Phys. Earth Planet. Inter. 42, 129-134 (1986).

19. Aldridge, K. D. \& Lumb, L. I. Inertial waves identified in the Earth's fluid outer core. Nature 325, 421 423 (1987).

20. Kobine, J. J. Inertial wave dynamics in a rotating and precessing cylinder. J. Fluid Mech. 303, 233-252 (1995).

21. Magaard, L. Ein Beitrag zur Theorie der internen Wellen als Störungen geostrophischer Strömungen. Deutsche Hydrogr. Z. 21, 241-278 (1968).

22. Cacchione, D. \& Wunsch, C. Experimental study of internal waves over a slope. J. Fluid Mech. 66, 223 239 (1974).

Acknowledgements. We thank G. van der Plas and S. Dalziel for help with post-processing of the data, and C. Staquet and B. Voisin for suggestions.

Correspondence should be addressed to L.R.M.M. (e-mail: maas@nioz.nl).

Discovery of a reactive azeotrope

\section{W. Song, R. S. Huss, M. F. Doherty \& M. F. Malone}

Department of Chemical Engineering, Goessmann Laboratory,

University of Massachusetts, Amherst, Massachusetts 01003-3110, USA

Mixtures are azeotropic if they can be distilled (or condensed) without a change of composition ${ }^{1}$. The existence of azeotropes in multicomponent mixtures in the absence of chemical reactions is well understood phenomenologically $y^{2,3}$ and theoretically ${ }^{4,5}$. Azeotropes place a fundamental limit on the compositions attainable in mixtures by fractional distillation, but they can in some cases be 'broken' by carrying out chemical reaction and separation simultaneously rather than sequentially ${ }^{6-9}$. Here we report the discovery of a boiling state of constant composition and temperature in a mixture of acetic acid, isopropanol, isopropyl acetate and water that is simultaneously in both reaction and phase equilibrium. These states, which we call reactive azeotropes, were predicted recently ${ }^{10,11}$. Without reaction, the mixture exhibits three twocomponent azeotropes, one three-component azeotrope but no four-component azeotrope; the last appears only under equilibrium reaction conditions. These findings may constrain technologies in which reaction and separation are conducted simultaneously, for example by limiting the conditions under which an azeotrope can be broken by chemical reactions to yield a high-purity product. In other cases the presence of a reactive azeotrope may be advantageous 9 .

Without chemical reaction, a mixture of acetic acid, isopropanol, isopropyl acetate and water has three binary azeotropes and a ternary azeotrope as listed in Table 1. There is no four-component azeotrope in this mixture. An acid-catalysed esterification reaction occurs in this mixture:

$$
\mathrm{CH}_{3} \mathrm{COOH}+\text { iso- } \mathrm{C}_{3} \mathrm{H}_{7} \mathrm{OH} \leftrightarrow \mathrm{CH}_{3} \mathrm{COOC}_{3} \mathrm{H}_{7}+\mathrm{H}_{2} \mathrm{O}
$$

This reaction occurs slowly; the rate can be increased substantially by the addition of a strong acid catalyst. The non-reactive binary azeotropes between water and isopropanol and between isopropyl acetate and isopropanol will also exist in the reacting mixture. However, a binary mixture of isopropyl acetate and water, as well as any mixture containing three of the components, will react and the corresponding azeotropes are absent.

A recent and general theory for mixtures in simultaneous vapour-liquid and chemical reaction equilibrium revealed the possibility of a state with a constant boiling temperature and constant, though not equal, vapour and liquid compositions ${ }^{10,11}$. In a reacting, boiling liquid each component has a rate of vaporization and a rate of creation or loss due to reaction. When the net rate for each component is zero, an azeotrope occurs. However, the existence of a reactive azeotrope has not been confirmed experimentally.

The theory ${ }^{10,11}$ provides a general transformation of variables 